\title{
A PRODUÇÃO DE IMAGENS PARA A PESQUISA EM GEOGRAFIA
}

\author{
- paulo cesar da costa GOMES \\ LETICIA PARENTE RIBEIRO
}

Resumo: Este artigo propõe uma reflexão acerca das relações entre as imagens e a geografia. Desde suas origens, o raciocínio geográfico esteve associado à produção de imagens, assumindo um verdadeiro imperativo gráfico. Neste sentido, parte da tradição geográfica concebe as imagens como instrumentos tanto de percepção como de compreensão do mundo, e não apenas como exemplos ou ilustrações. 0 campo de estudos empírico escolhido para desenvolver esta abordagem foi constituído pelos espaços públicos, os quais se apresentam à observação como uma sucessão de imagens em movimento, compondo quadros efêmeros, que denominamos comumente como a "cena pública". A investigação audiovisual sistemática desses lugares nos levou à consolidação de uma série de procedimentos metodológicos, centrados na reabilitação do papel da observação para a pesquisa geográfica.

Palavras-chave: Imagens; Espaços Públicos; Descrição; Metodologias Visuais

Introdução

Esse artigo tem um objetivo claro e preciso. Ele é um convite para pensarmos sobre as relações entre as imagens e a geografia.
Poucas vezes o fizemos. $\mathrm{Na}$ bibliografia geográfica, as poucas referências, quando aparecem, em geral, são subsidiárias de outras finalidades. Costumamos nos debruçar sobre imagens na Geografia quase exclusivamente quando desejamos ampliar a divulgação de certos conteúdos, quando as 
instrumentalizamos como ferramentas pedagógicas ou, ainda, quando as acusamos de veicular voluntariamente um conteúdo ideológico camuflado por trás das formas, dos traçados, das cores etc. (JAY, 1993; BOUSQUET-BRESSOLIER, 1995; HARLEY, 2001; FARINELLI, 2009).

Talvez pelo fato de termos chegado às imagens por uma via diversa, conduzidos pela pesquisa sobre os espaços públicos, nosso percurso seja bastante distinto desses que foram anteriormente elencados. Nosso primeiro interesse foi o de reconhecer o valor heurístico das imagens. Queríamos aprender com elas e, por isso, as tratamos não como algo subsidiário, mas como um elemento primário da pesquisa. É exatamente isso o que vamos apresentar nesse artigo.

De fato, seria injusto não reconhecer que, nos últimos dez anos, há um crescente interesse sobre as imagens por parte de alguns pesquisadores na Geografia (GODLEVSKA, 1999; ROSE, 2003，2006; DRIVER, 2003; RYAN，2003; THORNES, 2004; GOMES, 2007, 2008a, 2008b, 2009, 2010; COSGROVE, 1993, 2008; BERDOULAY e Saule-SORBÉ, 1998, 1999; BERDOULAY e GOMES, 2010; DANIELS et al., 2011). Esse despertar, de forma global, exprime o anseio de estabelecer a maneira pela qual as imagens colaboram nos procedimentos de construção do pensamento geográfico. De maneira mais específica, esse interesse é guiado segundo duas principais orientações.
A primeira, quase absoluta em número, reagrupa os estudos nos quais são diretamente procurados conteúdos geográficos nas imagens, tenham sido elas produzidas com esse fim ou não. Trata-se dos estudos que examinam pinturas, desenhos, fotografias, filmes, mas também mapas, cartogramas, gráficos etc., e procuram reconhecer a autoridade pedagógica e de comunicação desses instrumentos e meios (BESSE，2003; ROBIC, 1993，2000; MENDIBIL, 1993，1999，2006). É preciso distinguir que, na maior parte desses casos estudados, não é atribuído à ação de produzir imagens nenhum efeito direto sobre a produção do conhecimento em Geografia. Dessa forma, há uma imensa dissociação entre o momento de reflexão e a figuração dos fenômenos. O papel das imagens se restringe, assim, à função exemplar e ilustrativa.

A segunda orientação é aquela que é pretendida aqui. Parte-se da ideia de que as imagens participam diretamente na construção do pensamento geográfico; elas são, nesse sentido, instrumentos de descoberta. A principal finalidade é compreender como se desenvolve uma reflexão a partir das imagens ou junto com elas. Dito assim, parece que esse procedimento é original e inédito, mas, na verdade, é possível encontrar fortes raízes dele na história do pensamento geográfico e, sobretudo entre aqueles que se notabilizaram como grandes intelectuais da Geografia.

O raciocínio geográfico sempre esteve associado a um imprescindível aparelhamento visual, atendendo, desde seus primórdios, a um 
verdadeiro imperativo gráfico. Essa necessária associação surge mesmo na denominação da disciplina: Geo + grafia, contendo, assim, em seu próprio corpo, a concepção de informações que estão gravadas, inscritas. Essas informações se revelam à medida que se desvenda o código pelo qual elas se exprimem, ou seja, as regras que as erigem ${ }^{1}$. Já no programa pretendido pela Geografia ptolomaica, isso nos é indicado com absoluta clareza (JACOB, 1991; ALPERS, 1983). Assim, é possível afirmar que a informação geográfica foi, desde os seus primórdios, informação gráfica. Não há, por assim dizer, um conteúdo abstrato pensado antes $\mathrm{e}$, depois, traduzido em imagens. As imagens são instrumentos da reflexão geográfica, colaboram diretamente na produção das ideias.

Na longa história da Geografia, essas imagens foram mapas, desenhos, gravuras, pinturas, fotografias, blocos, diagramas, fluxogramas, esquemas, gráficos, tabelas, filmes etc, como nos foi dito em inúmeras oportunidades (BRUNET, 1980; HARLEY e WOODWARD, 1987; DANIELS, 1993; ANGOTTI-SALGUEIRO, 2005; CASTI, 2007; HERNANDO, 2009). Recentemente, com o desenvolvimento da computação e do sensoriamento remoto, assistimos à associação de bancos de dados com bases espaciais, gerando os assim denominados SIGs, que correspondem a uma nova geração de sistemas para reunir informações geográficas e, desse ponto de vista, são um patamar suplementar dessa longa história do desenvolvimento de sistemas de informação em Geografia (DENÈGRE e SALGÉ, 2004).

Imagens são constituídas pelo saldo das opções de seleção de critérios e variáveis que produzem formas e, através delas, destas formas, algo se torna visível. As imagens são artefatos que nos permitem ver determinadas coisas (MONDZAIN, 2003).

Voltamos, assim, ao fato de que imagens não são aqui concebidas como fenômenos que possam habitar uma dimensão puramente mental. Elas possuem sempre uma expressão física, um suporte pelo qual tomam forma, seja de natureza visual, seja auditiva. No extremo limite dessa argumentação, as imagens podem ser criadas textualmente (DUNCAN, 1990; MENDIBIL, 2000; PALSKY, 2010). Narrativas podem ser imagens, podem construílas e isso é verdadeiramente uma das características fundamentais e marcantes do discurso geográfico em diferentes momentos de sua evolução, (HUMBOLDT, 1808， 1810, 1848; VIDAL DE LA BLACHE, 1908). O exemplo da noção de paisagem é, nesse caso, bastante eloquente.

Hoje, comumente, nos referimos à paisagem como algo evidente $e_{i}$ utilizamos mesmo essa palavra como indicador dos elementos encontrados (visíveis) em uma esfera qualquer. Esse é o caso quando, por exemplo, nos referimos à "paisagem audiovisual" de um ambiente ou à "paisagem cultural" de um lugar ou de um momento na história. Entretanto, essa palavra não existia até pelo menos o final da Idade Média europeia (BERQUE, 1995). Se o 
conceito não existia, era porque aquilo que ele denomina e designa, ainda que não fosse de forma absoluta inexistente, era invisível aos olhos da época. O recorte de um ângulo de um lugar, de uma visada, tomada de um dado ponto de vista passou a nos fazer ver o desafio da transformação do espaço natural pelo trabalho humano. Esse ponto de vista se estetiza, ganha valor e se transforma em veículo de uma compreensão. Em outras palavras, percebemos, pela exposição do fragmento de um panorama, a importância da cultura na transformação do espaço. Por isso é justificável afirmar que as imagens são artefatos visuais que funcionam como instrumentos tanto de percepção como de compreensão do mundo.

Depois dessas breves considerações, já estamos mais à vontade para apresentar a forma pela qual as imagens se impuseram a nós como elementos essenciais da pesquisa em Geografia.

Durante os muitos anos que vimos estudando os espaços públicos, observamos a insistência pela qual esses espaços se apresentam a nós habitualmente e primariamente como uma sucessão de imagens em movimento. Essas imagens compõem um aglomerado de figuras, um quadro composto de eventos efêmeros, que denominamos comumente como a "cena pública" (GOMES, 2007). Dessa forma, essa cena, conjunto de muitas e variadas imagens sequenciadas, passou a ser vista por nós como um dos elementos de base na percepção que temos do que seria a vida pública. Partimos, portanto, da ideia de que vêla, observá-la, conhecê-la, tal qual ela se apresenta para nós habitualmente, seria um grande passo para poder compreendê-la e discuti-la.

Uma das primeiras constatações nas observações sobre esses espaços foi a leitura diferenciada que poderia ser feita seguindo os múltiplos lugares nos quais se estruturavam as cenas. $\mathrm{Na}$ verdade, munidos de uma sensibilidade geográfica, percebemos que comportamentos, gestos, maneiras de se apresentar, de falar, de estar nesses espaços produzem significações adjuntas à localização precisa do evento. Assim, obviamente, um mesmo gesto terá leituras e compreensão diversas segundo o lugar ao qual ele se associa.

Há toda uma tradição de análise sociológica, que se estende desde G. Simmel até a E. Goffman e a sociologia interacionista, passando pela assim denominada Escola de Chicago, que sempre foi muito sensível à espacialidade dos fenômenos sociais (GOFFMAN, 2010; FRISBY e FEATHERSTONE, 1997). Isso nos serviu como estrutura básica na interpretação das cenas que dão vida a esses espaços públicos. A leitura fina das dinâmicas sociais sobre eles se nutriu assim desse reconhecimento de uma associação essencial entre os comportamentos sociologicamente significativos e a orientação espacial dada pela maneira como se estruturam esses espaços.

Quando dizemos leitura fina, isso expressa a consideração da morfologia espacial em diferentes aspectos que vão desde o estatuto jurídico aos detalhes sobre os desníveis, 
revestimentos, condições de iluminação etc., sem esquecer os diferentes objetos (placas, cores, barreiras etc.) capazes de gerar indicações de sentidos para a mobilidade, de interdição, de condições de uso etc. Todo esse conjunto variado serve para classificar e diferenciar porções do espaço e o espaço serve por sua vez para classificar, ler e julgar os comportamentos que nele se passam.

Percebemos, assim, que os espaços públicos são um campo de práticas muito variadas. Comumente, eles abrigam atitudes esperadas ou passíveis de serem antecipadas como possibilidades, segundo os diferentes tipos de espaço. Trata-se de uma expectativa confortada por comportamentos mais ou menos ritualizados, conhecidos e aguardados. Há, no entanto, também uma imensa gama de transgressões que vão desde aquelas mais "toleráveis" aos olhos públicos até aquelas que ferem fortemente a vocação ou destinação desses espaços. Esses comportamentos e desvios podem, por um lado, constituir uma ameaça ao estatuto e à potência pública, mas podem também, por outro lado, ser o anúncio de mudanças na qualificação e classificação desses espaços que estão, aliás, sempre em mutação.

O intricado jogo nascido da associação entre "espécies de espaço", como poderia dizer Perec (1974), e todo um leque variado de comportamentos possíveis gera julgamentos, produz significações nos observadores. $\mathrm{O}$ olhar público é interpelado a todo instante pela cena urbana, pois toda ação que aí ocorre é pública, se faz sobre o olhar público, tem publicidade por isso gera julgamentos e significações. Mesmo aqueles que procuram atravessar esses espaços protegidos por carapaças de incomunicabilidade são objeto de observação e de interação. Denominamos de "reflexividade" essa propriedade dos espaços públicos de gerar uma infinita e recíproca cadeia de observadores. Notemos que essa interação é, antes de tudo, forjada em uma cena, a partir de imagens cruzadas. Essas imagens são, portanto, estruturantes dos espaços públicos e da vida pública.

Deduzimos dessa concepção de uma cena pública composta por uma contínua miríade de eventos que nossa percepção é necessariamente fragmentada, parcial e orientada. Tudo dependerá do lugar de onde observamos e da direção e foco do olhar, em suma, do ponto de vista ${ }^{2}$. Compreendemos, assim, facilmente que aquilo que se vê é apenas parte do que estaria visível sobre esse campo e que nossa orientação, posição ou interesse não pode tudo contemplar.

Um artifício capaz de potencializar a observação consiste, portanto, em multiplicar os pontos de vista. Outra possibilidade que pode ser somada a essa é alterar o nosso foco de atenção ou de interesse dentro de uma cena. Essas duas condições podem ser um pouco melhor satisfeitas se gravarmos cenas desse espaço a partir de diferentes ângulos, direções, e se deixarmos as cenas se desenvolverem para depois com elas discutirmos o que vemos. 
Logicamente, havia um ponto de partida na observação desses espaços. Nossa interrogação fundamental se dirigia à importância deles na constituição da vida social e democrática (GOMES, 2001; 2004). Essa interrogação, no entanto, estava, desde então, metodologicamente associada à maneira como essa vida se apresenta, se mostra nesses espaços. Em outras palavras, as imagens compõem esses espaços públicos; as cenas são a vida pública.

Já que o objeto fundamental do nosso interesse é a vida pública, com seus ritmos e seus pequenos rituais, a melhor escolha para a gravação foi deixar a câmera livremente capturar a banalidade do movimento que compõe o quadro habitual desses espaços. Decidimos também que, além disso, faríamos pequenas entrevistas com pessoas situadas nos próprios espaços públicos (em movimento, paradas em pé, sentadas, trabalhando etc.) e de diferentes categorias de idade, sexo, perfil sócioeconômico, e a elas perguntaríamos tão somente - o que é o espaço público? Além disso, tentamos variar os momentos do dia e os dias da semana. Começamos as entrevistas no Centro do Rio e nos deslocamos depois para os espaços da cidade que eram citados espontaneamente pelos entrevistados como exemplos.

A escolha dos lugares de filmagem se mostrou uma questão bastante complexa, similar àquelas que construímos comumente nas pesquisas de campo em Geografia. Escolher um lugar significa propor um ponto de vista, um enquadramento, uma escala de aproximação e uma composição. Essa escolha é uma colocação em relação das coisas que estão juntas, enquadradas e que produzem sentido uma vez que estão associadas pela posição no espaço.

Ao fim desse trabalho, tínhamos reunido uma grande quantidade de matéria fílmica e decidimos fazer uma montagem com a edição das imagens - um filme ${ }^{3}$. A ideia sempre foi a de fazer do espaço o tema e o personagem central. A câmera é o instrumento pelo qual observamos com apuro comportamentos, orientações, deslocamentos, interações etc., mas tudo isso é lido e interpretado em relação à espacialidade.

A edição procurou também, tanto quanto possível, não sugerir conclusões, não coordenar sentidos, não encaminhar pela montagem uma compreensão construída fora da imagem. Todo o projeto desse filme repousou na convicção da capacidade heurística das imagens (GOMBRICH, 1986). A projeção do filme renova, a cada visualização, a capacidade dessas imagens de produzir significações variadas, contraditórias, discutíveis; em suma, a cada projeção se estabelecem as condições de instaurar e renovar um debate em torno da interpretação de algo que se apresenta no momento em que se discute (LACERDA, 2011; MANGUEL, 2009).

Por isso, o filme não possui um caráter demonstrativo. Não é um filme documentário com a pretensão de construir a apresentação de uma tese que aparecerá ao final da projeção, costurada pelo encadeamento sequenciado das imagens. Não há uma exposição sobre o que são os espaços públicos. O filme e as imagens que o 
compõem são um meio de promover um debate, não um fim. Tudo o que vemos projetado não se destina a resolver um problema ou dar uma resposta sobre o que são esses espaços. Esse conjunto de imagens e cenas serve como meio de construir questões, de restabelecer o valor e o interesse de nossos gestos mais comuns e cotidianos em público, do peso das configurações espaciais nas nossas atitudes e na maneira como as qualificamos a partir dessa orientação espacial.

Concretamente, o filme é composto por uma sucessão de cenas habituais, ordinárias, em logradouros públicos na cidade do Rio de Janeiro. Por meio da projeção, essas cenas ganham distância em relação à nossa experiência mais imediata. Elas assim se transformam em objeto de reflexão, são tomadas como unidades de significação, ganham nova dimensão e estatuto. Elas se tornam extraordinárias quando extraídas do fluxo e da continuidade da vida urbana. A projeção das imagens amplia sempre a visibilidade de um fenômeno, atrai nossa atenção, interpela nossa compreensão (XAVIER, 2005). Trata-se de uma experiência estética e, como tal, convoca a sensibilidade, sem confundir inteiramente o visto com o vivido (TUAN, 1989). Experiências estéticas são reconhecidamente poderosos instrumentos de observação e análise (AUMONT et al., 2007).

Do ponto de vista da montagem, os planos são quase sempre fixos, o ritmo dado pela edição é movimentado, a qualidade das imagens não segue um padrão rígido e o som é captado diretamente. Não há nenhuma cena que não seja espontânea, não há na edição o recurso às oposições binárias, ao jogo de contrastes que força uma dada compreensão. Não há respostas anteriores às imagens e, por isso, elas não funcionam como um meio de ilustração ou exemplificação. Elas são um meio de observação e uma provocação ao debate. De fato, o filme pode muito bem ser visto em outra ordem, as cenas podem ser vistas separadamente, o espectador pode colocar seu interesse nos eventos que ocorrem nos limites do quadro. Nada disso compromete a ideia fundamental de provocar o debate a partir daquilo que vemos.

Nossa cultura visual comum, construída pela visualização de inúmeros filmes, construiu o hábito de encontrar uma coerência global nas sequências de imagens, uma trama que se resolve ao final da projeção. Isso muitas vezes nos leva a desejar que esses pequenos e múltiplos eventos apresentados nas cenas urbanas do filme construam uma trajetória que nos guie a um sentido ou compreensão global. Devemos, entretanto, convir que o fluxo da vida urbana não possui essa teleologia e que nossa percepção é sempre parcial e fragmentada. Por isso, todo sentido encontrado é sempre arbitrário e construído pela escolha da ordem e importância que damos a determinadas coisas, em meio a outras que ali também estão presentes. Não há, assim, um sentido oculto a ser revelado ao final da projeção. Toda compreensão é objeto de debate e a atualização pela apresentação das imagens restitui a possibilidade de discutir algo que se apresenta e 
estabelece a pluralidade de significações possíveis.

Ao final dessa experiência com o filme, sentimos que algo havia se consolidado no tratamento das imagens e em suas possibilidades dentro do campo da Geografia. Passamos, então, a pensar em desenvolver um conjunto de procedimentos mais sistemáticos para constituir uma verdadeira metodologia visual a ser usada em Geografia.

Essa metodologia se fundamenta no registro fílmico de planos-sequência de duração variável em espaços públicos. A opção por fazer planos-sequências encontra razão na unidade dramática e na continuidade de tempo e de espaço dentro de uma mesma cena (JULLIER, 2007). Assim, fica assegurado o fio narrativo da

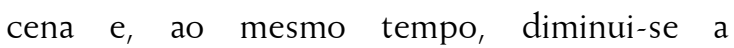
intervenção direta do realizador, uma vez que não há propriamente uma edição e os cortes são apenas aqueles que delimitam o começo e o fim da cena.

A experiência pioneira realizada na cidade do Recife, e comentada em trabalho anterior (GOMES e RIBEIRO, 2012), foi renovada uma dezena vezes com públicos diversos e em diferentes cidades ${ }^{4}$. Os resultados na interpretação do material gravado sempre foram muito ricos ${ }^{5}$. A capacidade de observar elementos e dinâmicas que não figuravam na intenção dos realizadores e a sensibilidade para perceber, ver e fazer associações ao serem projetadas as cenas sempre confirmaram o êxito da iniciativa. É, sem dúvida, impressionante a riqueza que têm as imagens de evocar múltiplas temáticas e discussões (AUMONT, 2005).

Essas experiências, além do rico material analítico produzido, foram apontadas também como geradoras de uma nova sintonia na observação das imagens. Muitos participantes das oficinas se disseram tocados por uma nova sensibilidade aos sons, cores, movimentos das pessoas, enquadramentos, composições e pelos detalhes na morfologia do espaço estampados nas imagens. Isso nos indica que há uma espécie de pedagogia, de educação visual geográfica, capaz de nos propiciar essa experiência de pensar com as imagens. Defendemos em outra oportunidade a ideia segundo a qual aquilo que vemos, como vemos e as condições que intervém sobre o que vemos definem diferentes regimes de visibilidade (GOMES, 2013). Analisar as imagens a partir de uma abordagem geográfica significa pensar sobre as condições que regulam nossa percepção sobre a espacialidade.

Outra importante contribuição decorrente destes trabalhos foi revisitar $\mathrm{O}$ estatuto da observação na pesquisa em Geografia, muitas vezes condenada, de forma rápida e negligente, a um lugar secundário nas reflexões de caráter teórico e metodológico do campo disciplinar.

No sentido de restituir à observação um papel central na pesquisa geográfica, optamos, tanto nas oficinas como em nossas experiências de produção audiovisual, pela adoção de um modelo pictórico capaz de evidenciar as condições subjacentes à própria construção das 
imagens. Partindo da classificação proposta pela historiadora da arte Svetlana Alpers (1983), elegemos a descrição como o modelo mais adequado para a investigação visual dos lugares que compunham nosso campo empírico de pesquisa. Ao estudar as principais escolas de pintura na Europa ocidental nos séculos XVI e XVII, a autora estabelece uma oposição entre os modelos narrativo e descritivo, o primeiro característico da pintura renascentista de matriz italiana e o segundo próprio da arte pictórica holandesa do período ${ }^{6}$.

Para Alpers (1983), a descrição é um modo de figuração de "mundos" tributário, em grande medida, dos modelos clássicos vinculados à cosmografia (como a geografia, a corografia e a topografia). Opõe-se à narração, pois, ao contrário desta, não parte de um texto anterior para produzir artefatos gráficos. A descrição não se restringe à enumeração exaustiva dos atributos de uma cena, lugar ou objeto, mas constitui uma verdadeira reflexão acerca das condições do olhar. Ao problematizar a exterioridade absoluta do ponto de vista que caracteriza a pintura narrativa clássica, fortemente associada ao desenvolvimento da perspectiva matemática, a pintura descritiva propõe um descentramento da observação. Em muitos casos, são figurados no interior do quadro o próprio autor da imagem (no caso, o pintor), além de outros observadores (personagens não envolvidos em qualquer ação significativa, e que apenas miram algum ponto situado dentro ou fora do quadro), os quais ampliam o campo de visão. Por estas e outras características, o modelo descritivo nos pareceu apropriado para a observação dos espaços públicos, tal como este conceito foi construído a partir de longos anos de reflexão acadêmica.

Uma vez feita a opção pela descrição, foi necessário tornar seus princípios operacionais para fins da pesquisa empírica e da concepção das oficinas. Para tanto, foram identificados alguns dispositivos construtivos básicos que caracterizam o modelo, os quais foram, então, aplicados à pesquisa de locação que antecedeu a realização do filme. Entende-se aqui a pesquisa de locação como uma investigação visual dos lugares, cujo objetivo é restituir a relação de interdependência entre os aspectos morfológicos e comportamentais que lhes são constitutivos. No caso específico dos espaços públicos, trata-se, além disso, de evidenciar os atributos que os diferenciam de outras classes de espaços, a saber, a copresença, a ordem e a reflexividade (GOMES, 2007).

As figuras abaixo resultam da aplicação prática de alguns dos dispositivos vinculados ao modelo descritivo. O primeiro, mais óbvio e largamente utilizado pela linguagem cinematográfica, consiste em interpelar um personagem com a câmera fazendo-o reconhecer a presença daquele que o observa (Figuras 1 e 2 ). Este dispositivo evidencia o caráter reflexivo não apenas da imagem, mas do próprio espaço público, tal como discutido anteriormente. A imagem é o resultado concreto deste encontro de olhares, e das 
diversas reações por ele suscitadas. Trata-se, portanto, de reconhecer a posição situada daquele que realiza o registro fotográfico ou fílmico.

Outro dispositivo, que também foi empregado na produção do filme, consiste em registrar a presença de outros observadores presentes no campo de visão da câmera (Figura 3). A simples presença destes múltiplos olhares no "interior" da cena obriga-nos a considerar aquilo que está "fora do campo" ou mesmo um elemento dentro do quadro que não havia sido notado, quer por aquele que produz a imagem, quer pela audiência. Este e outros recursos, como o utilizado na Figura 4 - que consiste no posicionamento da câmera em um ângulo que permite enquadrar um espaço liminar, de contato entre duas unidades morfológicas distintas -, acentuam um aspecto fundamental da produção imagética, qual seja, a permanente tensão entre ausência / presença que caracteriza os mundos figurados pela observação.

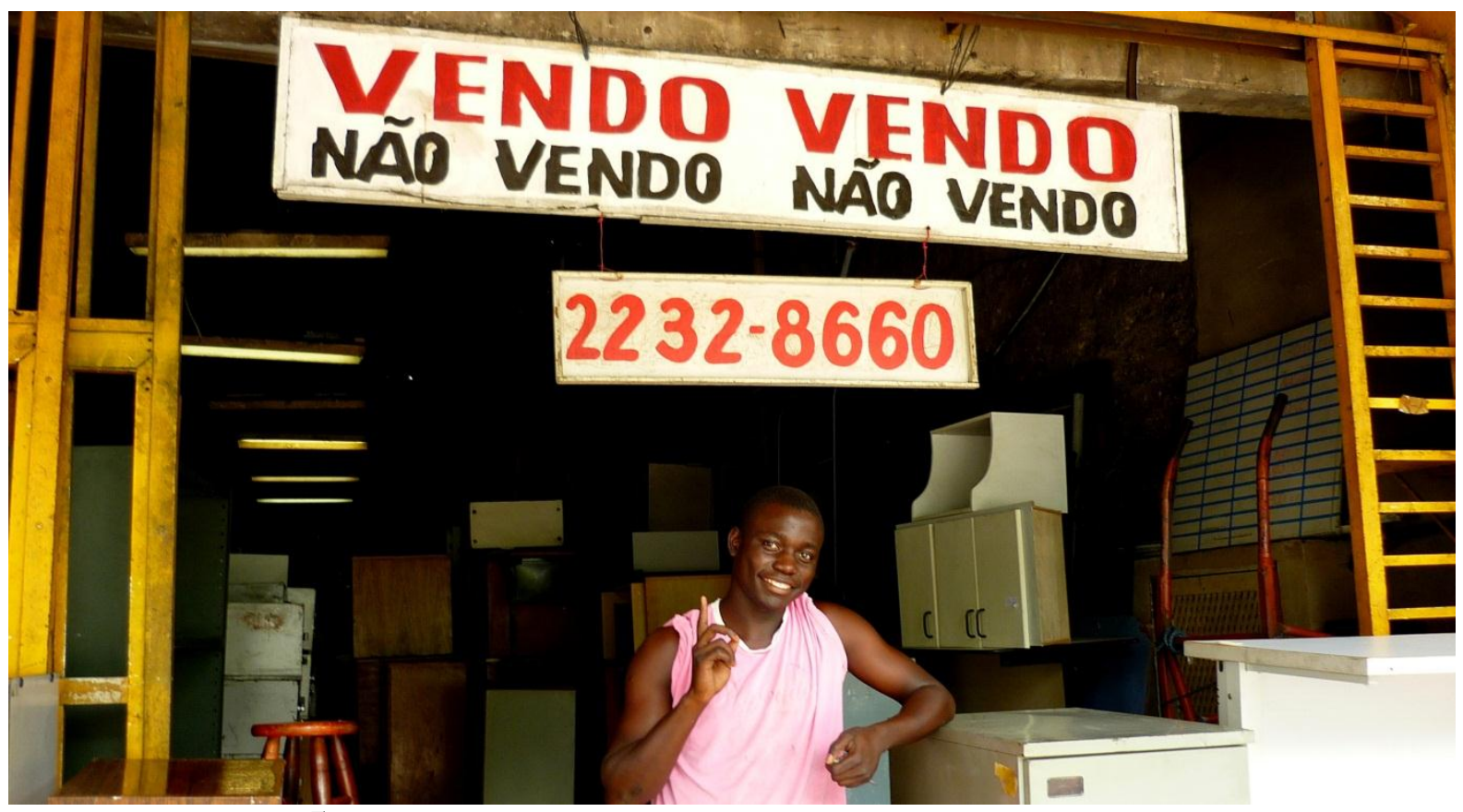

Figura 1: Reflexividade e publicidade (Foto: Leticia Parente Ribeiro, 2009) 


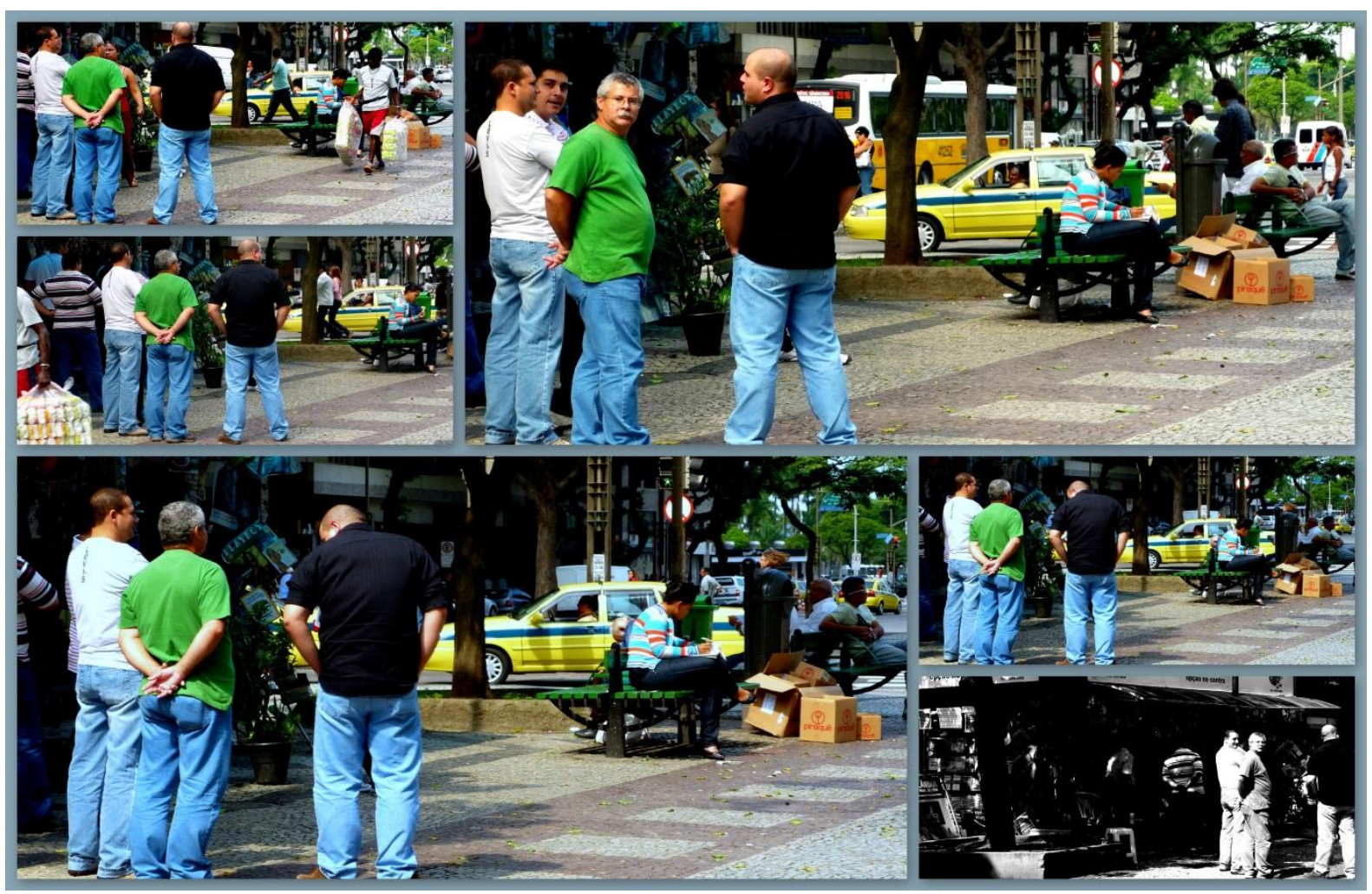

Figura 2: Encadeamento de olhares (Fotos: Leticia Parente Ribeiro, 2009)

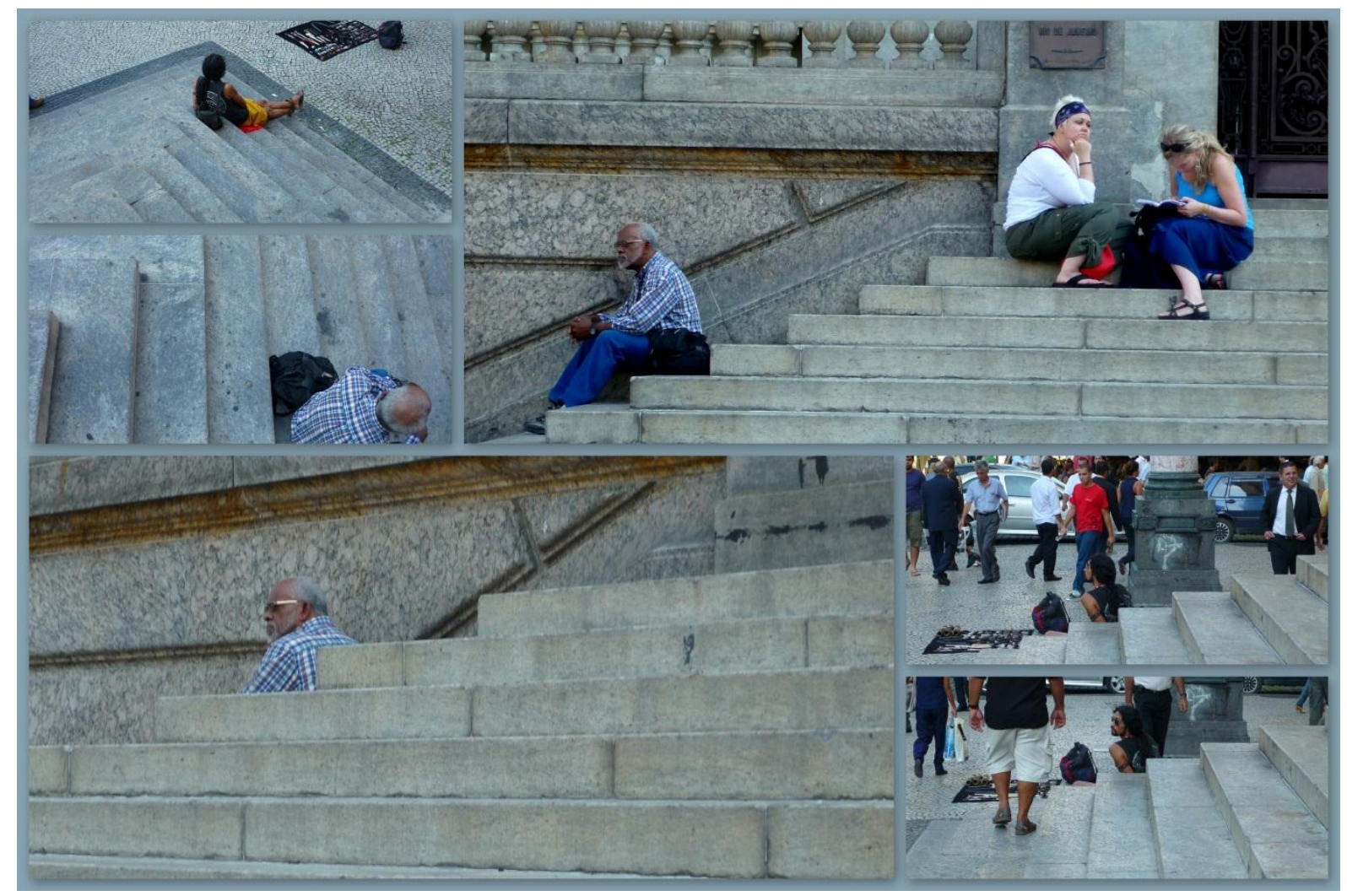

Figura 3: A multiplicação de olhares (Fotos: Leticia Parente Ribeiro, 2009) 

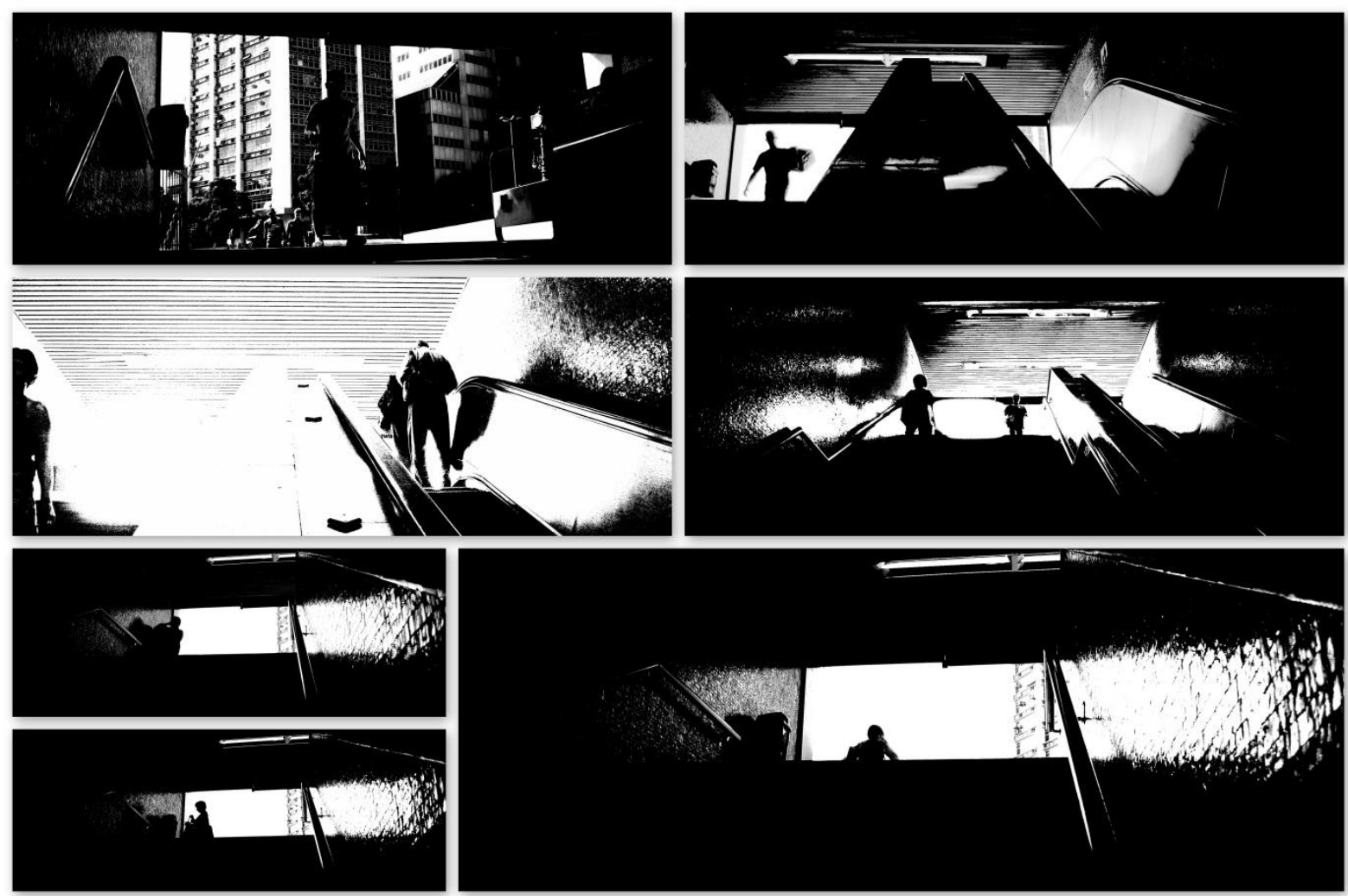

Figura 4: Presença / Ausência (Fotos: Leticia Parente Ribeiro, 2009)

A partir desta exposição, consideramos termos discutido os elementos básicos que compõem uma proposta (entre muitas outras possíveis) de metodologias visuais específicas para a pesquisa geográfica, uma abordagem mais

\section{NOTAS}

${ }^{1}$ Por isso grafia se traduz comumente como escrita.

${ }^{2}$ Sobre a natureza geográfica dessa noção de "ponto de vista", ver Gomes (2013).

3 Espaços Públicos: a cidade em cena. Acessível em: http://territorioecidadania.com/videos.

${ }^{4} \mathrm{O}$ público era sempre universitário, de Graduação e de PósGraduação, e as cidades foram: Rio de Janeiro, Recife, Florianópolis, Curitiba, Natal e Campinas, no Brasil e nas cidades de Pau, na França e Bogotá, na Colômbia.

${ }^{5}$ No site http://territorioecidadania.com/ é possível obter mais detalhes sobre essas oficinas. sensível à observação e à produção de imagens, ou seja, mais em sintonia com a sensibilidade gráfica que é parte da tradição deste campo do conhecimento.

\footnotetext{
${ }^{6}$ Para uma discussão acerca do papel da descrição em outras linguagens artísticas e mídias, ver MORETTI (2006); WOLF e BERNHART (2007)
}

\section{REFERÊNCIAS} BIBLIOGRÁFICAS

ALPERS, S. The art of describing: Dutch art in the seventeenth century. Chicago: The University of Chicago Press, 1983

ANGOTTI-SALGUEIRO, H. «A construção de representações nacionais: os desenhos de Percy Lau na Revista Brasileira de Geografia e outras "visões iconográficas" do Brasil moderno ». Anais do Museu Paulista: História e Cultura Material, 13 (2), 2005, p. 21-72.

AUMONT, J. L'image. Paris: Armand Colin, 2005 
AUMONT, J. et al. A Estética do filme. São Paulo: Papirus Editora, 2007.

AUSTER, P. Auggie Wren's Christmas Story. New York: Henry Holt and Co, 2004

BERDOULAY, V. ; SAULE-SORBE, H. «La mobilité du regard et son instrumentalisation. Franz Schrader à la croisée de l'art et de la science ». Finisterra, 38 (65), 1998, p. 39-50.

BERDOULAY, V. ; SAULE-SORBE, H. «Franz Schrader face à Gavarnie, ou le géographe peintre de paysage ». Mappemonde, 55 (3), 1999, p. 33-37.

BERDOULAY, V.; GOMES, P. C. C. «Introduction: Image et espace public: la composition d'une scène ». Géographie et Cultures, Paris, 73, 2010, p. 3-6.

BERQUE, A. Les raisons du paysage. Paris: Hazan, 1995.

BESSE, J.-M. Face au monde. Atlas, jardins, géoramas. Paris: Desclée de Brouwer, 2003.

BOUSQUET-BRESSOLIER, C. (dir). L'oeil du cartographe. Paris: CTHS, 1995.

BROC, N. La géographie des Philosophes. Géographes et voyageurs du XVIIIe siècle. Paris: Ophrys, 1975.

BRUNET, R. « La composition des modèles en analyse spatiale ». L'Espace géographique, 9, 1980, p. 253-265.

CASTI, E. (dir). Cartografia e progettazione territoriale: dalle carte coloniali alle carte di piano. Turin: Utet, 2007.

COSGROVE, D. Social formation and symbolic landscape. Madison: University of Wisconsin Press, 1993.

COSGROVE, D. Geography and vision. Londres: I.B. Tauris, 2008.

DANIELS, S. Fields of vision. Landscape imagery and national identity in England and Wales. Princeton: Princeton University Press, 1993.

DANIELS, S. DE LYSER, D. ENTRIKIN, N.; RICHARDSON, D. (eds.). Envisioning Landscapes, Making Worlds: Geography and the Humanities. London: Routledge, 2011.

DENEGRE, J.; SALGE, F. Les systèmes d'information géographique, Paris: PUF, 2004

DRIVER, F. «On Geography as a visual discipline ». Antipode, 2003, p. 227-231

DUNCAN, J. The city as text: The politics of landscape interpretation in the Kandyan kingdom, Cambridge: Cambridge University Press, 1990

FARINELLI, F. De la raison cartographique, Paris: CTHSEditions, 2009

FRISBY, D.; Featherstone, M. Simmel On Culture: Selected Writings Theory, Culture \&Society. Nottingham: Sage, 1997.

GODLEWSKA, A. «From enlightenment vision to modern science? Humboldt's visual thinking ». In: Livingstone D. ; Whiters C. (eds.). Geography and Enlightenment. Chicago: Chicago University Press, 1999, p. 236-279.

GOFFMAN, E. Comportamento em lugares públicos. Petrópolis: Editora Vozes, 2010
GOMBRICH, E. H. Arte e Ilusão: Um estudo de psicologia da representação pictórica. São Paulo: Martins Fontes, 1986

GOMES, P. C. C. «L'espace public métropolitain et le recul de la culture civique: l' exemple Du Brésil ». In: GHORRA-GOBIN, C. (dir.). "Réinventer Le sens de la ville: Les espaces publics à l'heure globale". L'Harmattan: Paris, 2001.

GOMES, P. C. C. «O silêncio das cidades: os espaços públicos sob ameaça, a democracia em suspensão ». Cidades, 2 (4), 2004, p. $249-266$

GOMES, P. C. C. «Cenários para a Geografia: Sobre a espacialidade das imagens $\mathrm{e}$ suas significações ». In: ROSENDAHL, Z.; CORRÊA, R. (Org.). Espaço e Cultura: pluralidade temática. Rio de Janeiro: EDUERJ, 2007, p. 187-210.

GOMES, P. C. C. «A cidade em imagens». Cidades, n ${ }^{\circ}$ temático, 1, 2008a.

GOMES, P. C. C.; GOIS, M. P. F. «A cidade em quadrinhos: elementos para a análise da espacialidade nas histórias em quadrinhos ». Cidades, 5 (7), 2008b, p. 17-32

GOMES, P. C. C. «Trois images, trois scénarios, un lieu: des Français à Rio de Janeiro ». In : GUICHARNAUD-TOLLIS, M. et al. (Org.). Regards Croisés entre la France et le Brésil. Paris: Harmattan, 2009, p. 19-42.

GOMES, P. C. C.; FORT-JACQUES, T. «Spatialité et portée politique d'une mise en scène: le cas des tentes rouges au long du Canal Saint-Martin », Géographie et Cultures (Paris), v. 1, 2010, p. 7-22.

GOME, P. C. C. ; Ribeiro, L. «Metodologias visuais e imaginação urbana: uma experiência no Recife ». In : MACIEL, C.A.; GONÇALVES, C. U. ; PEREIRA, M. C. Abordagens geográficas do urbano e do agrário. Recife : Ed. Univesitária da UFPE, 2012, p. 63-73.

GOMES, P. C. C. O lugar do olhar: Elementos para uma geografia da visibilidade. Rio de Janeiro: Bertrand Brasil, 2013.

HARLEY, J. B. The new nature of maps. Essays in the history of cartography. Baltimore: Johns Hopkins Press, 2001

HARLEY, J. B.; WOODWARD, D. (dir.). Cartography in Prehistoric, Ancient, and Medieval Europe and the Mediterranean. Chicago: University of Chicago Press, v. 1, 1987.

HERNANDO, A. «Retórica iconográfica e imaginación geográfica: los frontispicios de los atlas como proclamaciones ideológicas », Boletín de la Asociación de geógrafos españoles, 51, 2009, p. 353-369

HUMBOLDT, A. Von. Tableaux de la nature. Paris : F. Schœell, 1808.

HUMBOLDT, A. Von. Cosmos. Essai d'une description physique du monde. Paris: Gide et Baudry, t. I, 1848

HUMBOLDT, A. Von., Vues des cordillières et monuments des peuples indigènes de l'Amérique (com apresentações de C. Minguet e J.-P. Duviols). Nanterre: Erasme, 1989 [1810].

JACOB, C. Géographie et ethnographie en Grèce ancienne. Paris: Armand Colin, 1991 
JAY, M. Downcast eyes. The denigration of vision in Twentiethcentury French thought. Berkeley: University of California Press, 1993.

JULLIER, L. L’analyse de séquences. Paris: Armand Colin, 2007.

LACERDA, T. A interpretação da imagem: subsídios para o ensino de arte. Rio de Janeiro: Mauad, 2011

MANGUEL, A. Lendo Imagens, São Paulo, Companhia das Letras, 2009

MENDIBIL, D. «Jean Brunhes, photographe-iconographe »e «Deux 'manières': Jean Brunhes et Paul Vidal de la Blache ». In : Autour du Monde. Jean Brunhes. Regards d'um géographe/regards de la géographie. Boulogne: Musée Albert Kahn, 1993, p. 140-157.

MENDIBIL, D. «Essai d'iconologie géographique ». L'Espace géographique, 4, 1999, p. 327-336.

MENDIBIL, D. « Paul Vidal de la Blache, le 'dresseur d'images'. Essai sur l'iconographie de La France. Tableau géographqiue (1908) ». In: ROBIC, M.-C. (dir.). Le Tableau de la Géographie de la France de Paul Vidal de la Blache. Dans le Labyrinthe des formes. Paris: CTHS, 2000, p. 77-105.

MENDIBIL, D. «O sistema iconográfico da geografia clássica francesa e Pierre Monbeig ». In: ANGOTTI-SALGUEIRO, $\mathrm{H}$. (org.). Pierre Monbeig e a Geografia Humana Brasileira. São Paulo: EDUSC, 2006, p. 235-250.

MONDZAIN, M.-J. Le commerce des regards. Paris: Seuil, 2003.

MORETTI, F. «Serious century ». In: MORETTI, F. (Ed.): The Novel, Volume 1 - History, geography and culture. Princeton: Princeton University Press, 2006, p.364-400.

PALSKY, G. «Le Tableau de la hauteur des montagnes. Un paysage de fantaisie entre art et géométrie ». In: ORTEGA, N.;
GARCIA, J.; RUIZ-GOMEZ, M. (dir.). Lenguajes y visiones del paisaje y del terirtorio. Madrid: UAM, 2010, p. 297-307.

PEREC, G. Espèces d'espaces. Paris: Galilée, 1974.

ROBIC, M.-C. «Jean Brunhes un 'géo-photo-graphe' expert aux Archives de la Planète». In : Autour du Monde. Jean Brunhes. Regards d'un géographe/regards de la géographie. Boulogne: Musée Albert Kahn, 1993, p. 109-137.

ROBIC, M.-C. (dir.). Le Tableau de la géographie de la France de Paul Vidal de la Blache. Dans le labyrinthe des formes. Paris: CTHS, 2000

ROSE, G. «On the Need to Ask How, Exactly, Is Geography "Visual"? ». Antipode, 35 (2), 2003, p. 212-221.

ROSE, G. Visual methodologies: an introduction to interpreting visual materials. Londres: Sage, 2006.

RYAN, J. «Who's affraid of visual culture ? ». Antipode, 35 (2), 2003, p. 232-237.

THORNES, J. «The Visual Turn and Geography (response to Rose 2003 intervention)». Antipode, 2004, p.787-794.

TUAN, Y.-F. «Surface phenomena and aesthetic experience ». Annals of the Association of American geographers, 79, 1989, p. 233-241.

VIDAL DE LA BLACHE, P. La France. Tableau géographique. Paris: Hachette, 1908.

WOLF, W.; BERNHART, W. (Eds.). Description in literature and other media. New York : Rodopi, 2007.

XAVIER, I. O discurso cinematográfico - a opacidade e a transparência. São Paulo: Paz e Terra, 2005. 
THE PRODUCTION OF IMAGES FOR RESEARCH IN GEOGRAPHY

ABSTRACT: THIS ARTICLE PROPOSES A REFLECTION ON THE RELATIONSHIPS BETWEEN IMAGES AND GEOGRAPHY. SINCE ITS ORIGINS, GEOGRAPHIC REASONING WAS ASSOCIATED WITH THE PRODUCTION OF IMAGES, ASSUMING A REAL GRAPHIC IMPERATIVE. IN THIS SENSE, PART OF THE TRADITION CONCEIVES GEOGRAPHIC IMAGES AS INSTRUMENTS OF BOTH PERCEPTION AND UNDERSTANDING OF THE WORLD, AND NOT ONLY AS EXAMPLES OR ILLUSTRATIONS. THE EMPIRICAL CASE STUDY CHOSEN TO DEVELOP THIS APPROACH WAS THE PUBLIC SPACES, WHICH PRESENT THEMSELVES TO OBSERVATION AS A SUCCESSION OF MOVING IMAGES, COMPOSING EPHEMERAL FRAMES, WHICH WE CALL COMMONLY AS THE "PUBLIC SCENE". THE AUDIO-VISUAL SYSTEMATIC RESEARCH ON THESE PLACES LED TO THE CONSOLIDATION OF A NUMBER OF METHODOLOGICAL PROCEDURES, FOCUSING ON THE REHABILITATION OF THE ROLE OF OBSERVATION FOR GEOGRAPHICAL RESEARCH.

KEYWORDS: IMAGES; COMMONS; DESCRIPTION; VISUAL METHODOLOGIES

LA PRODUCTION D'IMAGES POUR LA RECHERCHE EN GÉOGRAPHIE

RESUMÉ: CET ARTICLE PROPOSE UNE RÉFLEXION SUR LES RELATIONS ENTRE LES IMAGES ET LA GÉOGRAPHIE. DEPUIS SES ORIGINES, LE RAISONNEMENT GÉOGRAPHIQUE A ÉTÉ ASSOCIÉE À LA PRODUCTION D'IMAGES, EN SUPPOSANT UN VÉRITABLE IMPÉRATIF GRAPHIQUE. EN CE SENS, UNE PARTIE DE LA TRADITION CONCOOIT IMAGES GÉOGRAPHIQUES COMME INSTRUMENTS À LA FOIS LA PERCEPTION ET LA COMPRÉHENSION DU MONDE, ET PAS SEULEMENT COMME DES EXEMPLES OU DES ILLUSTRATIONS. LE CHAMP D'ÉTUDES EMPIRIQUES CHOISI DE DÉVELOPPER CETTE APPROCHE A ÉtÉ FAITE PAR LE PUBLIC, QUI SE PRÉSENTENT À L'OBSERVATION COMME UNE SUCCESSION D'IMAGES EN MOUVEMENT, LA COMPOSITION DES CADRES ÉPHÉMÈRES QUE NOUS APPELONS COMMUNÉMENT LE «SCÈNE PUBLIC》. LA RECHERCHE AUDIOVISUEL SYSTÉMATIQUUE DANS CES LIEUX A CONDUIT À LA CONSOLIDATION D'UN CERTAIN NOMBRE DE PROCÉdURES MÉtHODOLOGIQUES, EN METTANT L'ACCENT SUR LA RÉHABILITATION DU RÔLE DE L'OBSERVATION POUR LA RECHERCHE GÉOGRAPHIQUE.

MOTS-CLÉS: IMAGES; COMMUNES; DESCRIPTION; MÉTHODOLOGIES VISUELS 
ESPAÇO E CULTURA, UERJ, RJ, N. 33, P.27-42, JAN./JUN. DE 2013 http://www.e-publicacoes.uerj.br/index.php/espacoecultura/ 